\title{
INOVAÇÃO COLABORATIVA: UMA ABORDAGEM ABERTA NO DESENVOLVIMENTO DE NOVOS PRODUTOS
}

\author{
COLLABORATIVE INNOVATION: AN OPEN APPROACH IN THE DEVELOPMENT OF NEW \\ PRODUCTS
}

INNOVACIÓN COLABORATIVA: UN ABORDAJE ABIERTO EN EL DESARROLLO DE NUEVOS PRODUCTOS

\section{RESUMO}

As estratégias que as empresas têm adotado para promover inovações tecnológicas de produtos e serviços vêm mudando significativamente nos últimos anos. Um dos elementos de notável evolução diz respeito à maneira como as empresas conduzem a etapa de pesquisa e desenvolvimento (P\&D) de novos produtos, serviços, processos e modelos de negócios. Diante dessa problemática, o presente artigo busca identificar práticas de inovação colaborativa no desenvolvimento de novos produtos. Para melhor compreender a mudança nos processos de inovação, elegeu-se como campo empírico um caso da indústria automotiva. Mesmo que essa indústria tenha experimentado o desenvolvimento de P\&D em conjunto com os seus fornecedores, a cocriação de valor com os usuários finais é algo muito novo. O objeto de análise foi o projeto de desenvolvimento do Fiat Mio - Fiat Concept Car III (FCCIII) e os resultados demonstraram que, durante o processo de desenvolvimento do FCCIII, foram adotadas diferentes práticas de inovação colaborativa, o que possibilitou o acesso de diferentes conhecimentos externos à organização.

PALAVRAS-CHAVE Inovação, colaboração, cocriação, indústria automotiva, inovação aberta.

Bruna Bueno bruninhabueno@gmail.com

Mestranda em Política Científica e Tecnológica pelo Instituto de Geociências, Universidade Estadual de Campinas - Campinas - SP, Brasil

Alsones Balestrin abalestrin@unisinos.br

Professor do Programa de Pós-Graduação em Administração, Universidade do Vale do Rio dos Sinos - São Leopoldo - RS, Brasil

Abstract The strategies adopted by companies seeking to foster technological innovations in products and services have been changing significantly in the last years. One of the elements of notable evolution is related with the way companies conduct the research \& development $(R \& D)$ of new products, services, processes and business models. The present article aimed to identify practices of collaborative innovation that have been used in the development of new products and elected a case from the automotive industry as its empirical field. Even though this industry has experienced the development of R\&D together with its suppliers, co-creating value with end-users is new. The unity of analysis was the development project of Fiat Mio - Fiat Concept Car III (FCCIII) and the results showed that, during the FCCIII's development process, different practices of collaborative innovation were adopted, which provided the focal organization with access to a different pool of knowledge, external to its boundaries.

keywords Collaboration, innovation, automotive industry, co-creation, open innovation.

Resumen Las estrategias que las empresas han adoptado para promover innovaciones tecnológicas de productos y servicios están cambiando significativamente en los últimos años. Uno de los elementos de notable evolución se refiere a la manera cómo las empresas conducen la etapa de investigación y desarrollo (P\&D) de nuevos productos, servicios, procesos y modelos de negocios. Frente a esa problemática, el presente artículo busca identificar prácticas de innovación colaborativa en el desarrollo de nuevos productos. Para comprender mejor el cambio en los procesos de innovación, se eligió como campo empírico un caso de la industria automotriz. Aunque dicha industria haya experimentado el desarrollo de P\&D en conjunto con sus proveedores, la cocreación de valor con los usuarios finales es algo muy nuevo. El objeto de análisis fue el proyecto de desarrollo del Fiat Mio - Fiat Concept Car III (FCCIII) y los resultados demostraron que durante el proceso de desarrollo del FCCIII se adoptaron diferentes prácticas de innovación colaborativa, lo que posibilitó el acceso de diferentes conocimientos externos a la organización.

Palabras clave Innovación, colaboración, cocreación, industria automotriz, innovación abierta. 


\section{INTRODUÇÃO}

As empresas organizam processos para a geração de inovação com o intuito de lançar novos produtos e serviços, desenvolver novos processos ou novas configurações organizacionais e atuar em novos mercados. Ao estudar o processo de inovação, autores como Clark e Wheelwright (1993) e Dodgson, Gann e Salter (2006) salientam que, durante muito tempo, esse processo foi desenvolvido com foco demasiado nos recursos internos, havendo pouca interação e baixo acesso ao conhecimento externo. Conforme destaca Rothwell (1995), os primeiros estágios do processo de inovação tecnológica, alcançados até a década de 1980, foram realizados de maneira eminentemente interna à empresa para gerar o conhecimento, desenvolver o produto e comercializá-lo no mercado.

Outros autores, entretanto, como Richardson (1972), Powell, Koput e Smith-Doerr (1996), Hage e Hollingsworth (2000), destacam a crescente complexidade tecnológica no desenvolvimento de novos produtos, o que vem instando as empresas à necessidade de acesso a conhecimentos externos por meio de relacionamentos colaborativos com outros agentes. No início desta última década, Chesbrough (2003) denominou essa mudança de perspectiva com o termo "inovação aberta" em alternativa ao modelo dominante "inovação fechada", representando, obviamente, dois extremos de um contínuo. Para o autor, a abertura do modelo fechado de inovação é imperativa, sobretudo pelo aumento da velocidade de lançamentos de novos produtos, ocasionado pela crescente redução dos seus ciclos de vida (CHESBROUGH, VANHAVERBEKE e WEST, 2006). Ao abrir-se para o ambiente externo, a empresa poderá acessar relevantes conhecimentos para o processo de inovação, especialmente dos consumidores, conforme evidenciado nos trabalhos sobre inovação democrática (VON HIPPEL, 2005), cocriação de valor (ENKEL e outros, 2009), consumidores criativos (BERTHON e outros, 2007) e colaboração em massa (POETZ e SCHREIER, 2012).

Em indústrias intensivas em conhecimento, as práticas de inovação colaborativa já ocorrem há algum tempo, como é o caso das indústrias de biotecnologia (POWELL, 1998) ou de produtos eletrônicos (DITTRICH e DUYSTERS, 2007). Ainda são pouco conhecidas na literatura, no entanto, as práticas colaborativas de inovação em indústrias mais maduras, como no caso do desenvolvimento de produtos na indústria automotiva. A tradição dessa indústria está no desenvolvimento de produtos por meio de alto nível de internalização, em especial devido aos altos investimentos em ativos específicos na produção de componentes constituintes de um veículo (CLARK e FUJIMOTO, 1991).

Em outros estudos, porém, Fujimoto e Takeishi (2001) demonstram que as empresas automotivas começaram a estabelecer parcerias para o desenvolvimento de tecnologias com seus fornecedores, dando os primeiros passos à abertura do processo de inovação. Evidências dessa perspectiva mais colaborativa de interação também podem ser encontradas no trabalho de Dyer e Nobeoka (2000) ao apresentar o sistema "Toyota" de relacionamento com sua rede de fornecedores e na pesquisa de Ili, Albers e Miller (2010), que apresentam inovação aberta na indústria automotiva alemã. Em um estudo comparativo em empresas japonesas e alemãs, Wilhelm (2011) busca identificar o nível de cooperação na cadeia de fornecimento, evidenciando que empresas como Toyota e BMW atuam com forte nível de cooperação com seus fornecedores e empresas como Nissan, Volkswagen e Honda, com nível de cooperação menos significativo.

Mesmo com a existência de estudos que trazem evidências de cooperação na indústria automotiva, geralmente essas relações ocorrem dentro da cadeia de fornecedores e no contexto de economias desenvolvidas. Carece, então, de estudos que apontem outros níveis de cooperação, como a cooperação com usuários finais, universidades e outros atores, no contexto de economias emergentes. Para o melhor entendimento acerca dessa problemática, o presente artigo pretende identificar práticas de inovação colaborativa no desenvolvimento de um novo produto no contexto da indústria automotiva brasileira. Diante da necessidade de delimitação da unidade de análise, a pesquisa se concentrará no estudo de caso do projeto de desenvolvimento do Fiat Mio (Fiat Concept Car III). A escolha empírica justifica-se pela singularidade do caso e por trazer práticas avançadas de inovação em uma empresa localizada em uma economia emergente. De acordo com Figueiredo (2004), para tornarem-se competitivas essas empresas têm que se engajar em um processo de aprendizagem tecnológica.

Para apresentar as reflexões teóricas e as evidências empíricas de modo a facilitar a compreensão do leitor, o artigo está estruturado da seguinte forma: inicialmente, apresentam-se as teorizações sobre o campo de estudo, abordando-se os conceitos de inovação, processo de desenvolvimento de novos produtos e inovação aberta 
e colaborativa. Na sequência, explica-se a metodologia de coleta e análise dos dados. Depois, descrevem-se os resultados do estudo de caso e, por fim, apresentam-se as considerações finais e os encaminhamentos para pesquisas futuras.

\section{INOVAÇ̃̃O NO CONTEXTO DAS ORGANIZAÇÕES}

Desde os estudos de Schumpeter (1934), a inovação é vista como fonte fundamental para a geração de vantagem competitiva, desenvolvimento econômico e mudanças na sociedade. Segundo Freeman (2003), quando a vantagem competitiva provém de uma mudança expressiva em um produto, serviço ou processo, ela é mais significativa. Tais inovações abrangem: a) introdução de um novo bem ou de uma nova qualidade de certo bem; b) introdução de um novo método de produção; c) abertura de um novo mercado para uma indústria; d) utilização de uma nova fonte de matéria-prima ou produto semiacabado e e) estabelecimento de uma nova organização em determinada indústria (NAPOLEONI, 1963). Dosi (1988) retoma o conceito de Schumpeter e refere-se à inovação como resultado dos processos de pesquisa, descoberta, experimentação, desenvolvimento, imitação e adoção de novos produtos, novos processos de produção e novas formas de organizações.

DeBresson (1997) argumenta que o desenvolvimento tecnológico avança graças à complementaridade de dois tipos de conhecimento: a) conhecimento técnico ou produtivo - geralmente específico e comunicado por meio de técnicas, práticas e regras preestabelecidas, ele advém de experiências e habilidades específicas (know-how); b) conhecimento científico - geralmente mais universal - emerge tanto dos conhecimentos humanos previamente acumulados quanto das experiências humanas. Cabe salientar que a transferência desses conhecimentos necessários à inovação não é um processo simples, unidirecional, ocorrido em um período de tempo determinado. Ao contrário, segundo Mowery e Rosenberg (1989), esse processo é complexo e interativo, pois o fluxo de informações ocorre em duas vias e é concebido como uma contínua atividade de pesquisa estruturada pelas forças econômicas, pelo conhecimento tecnológico e pela demanda dos consumidores por diferentes categorias de produtos e serviços.
Para compreender melhor o atual modelo de inovação junto às organizações, é preciso observar a sua evolução histórica. Para Rothwell (1995), o modelo de inovação atual corresponde a um modelo de sistemas abertos e integrados em rede. A tendência é que as equipes de P\&D trabalhem integradamente e realizem ações colaborativas entre diversos agentes, tanto no sentido vertical da cadeia quanto no sentido horizontal (entre empresas de um mesmo segmento). Dessa maneira, o resultado da inovação passa a ser uma ação conjunta e cooperada entre diversos atores internos e externos à organização, como empresas, fornecedores, clientes, além de outras instituições de caráter público ou privado (O'CONNOR, 2006).

De modo geral, as tecnologias demandam o uso simultâneo de diferentes conjuntos de habilidades e bases de conhecimentos em um processo de inovação, que está limitado a uma empresa individual (POWELL, KOPUT e SMITH-DOERR, 1996). A colaboração em rede facilita a reunião de complementaridade de habilidades de diferentes firmas (RICHARDSON, 1972). Hage e Hollingsworth (2000), no entanto, salientam que muitas das pesquisas têm enfocado somente as características organizacionais internas que afetam as taxas de inovação, ignorando os aspectos externos, tal como salientado por Powell (1998), quando o autor destaca que a colaboração interorganizacional proporciona melhores resultados de inovação em relação às firmas que trabalham individualmente.

Assim, torna-se evidente que as relações colaborativas interfirmas podem viabilizar o acesso a uma grande quantidade de conhecimento para os processos de inovação (HUIZINGH, 2011), permitindo à empresa abrir-se para novas ideias do ambiente externo (REED, STORRUD-BARNES e JESSUP, 2012) e avançar para modelos de desenvolvimento em conjunto de P\&D e novas práticas de cocriação de valor (HUSTON e SAKKAB, 2006). Essa abordagem aberta e colaborativa de inovação será aprofundada na sequência do trabalho.

\section{PROCESSO ABERTO E COLABORATIVO NA GERAÇÃO DE INOVAÇÃO}

O processo de geração de inovação em produtos e processos costuma ser representado pelo modelo stage gate (COOPER, 1990) ou pelo modelo funil de desenvolvimento (CLARK e WHEELWRIGHT, 1993). 
Ambos sustentam que o processo de inovação seja dividido em estágios (stage) e que, ao fim de cada um desses estágios, haja um "portão de decisões" (gate) para avaliar quais projetos devem ser continuados ou descontinuados. Uma questão colocada na literatura é que esses estágios são desenvolvidos, tradicionalmente, pelo P\&D interno da empresa, com baixa interação e colaboração com atores externos, sendo denominado por Chesbrough (2003) como modelo de inovação fechada. No entanto, "[...] a inovação não é gerada somente por recursos que uma empresa é capaz de desenvolver internamente, mas também através do acesso de recursos e de capacidades de organizações externas, que a empresa pode acessar por meio de alianças e de acordos de cooperação" (SODA, 2011, p. 661).

Segundo Tidd, Bessant e Pavitt (2001), explorando recursos de outras organizações externas uma empresa consegue reduzir os custos de desenvolvimento tecnológico, os riscos de entrada no mercado e o tempo de desenvolvimento de um novo produto. Essa perspectiva, denominada por Chesbrough (2003) como modelo de inovação aberta (Figura 1), sustenta que o processo de inovação deva ser mais colaborativo, buscando acessar conhecimento a partir de diversos atores externos. Da mesma maneira, o conhecimento pode fluir para fora da organização por meio de licenciamentos, tecnologias e spin-offs. O resultado pode ser um novo produto desenvolvido para o mercado atual da empresa, um novo produto para um novo mercado ou ainda um produto para ser integrado ao portfólio de outra empresa por meio do licenciamento de patentes.

Estudos empíricos, como os de Van der Meer (2007), identificam que, na fase inicial de desenvolvimento do conceito do produto, as empresas acessam principalmente ideias por meio de conferências, feiras, contatos com fornecedores, clientes e relações com universidades e institutos de pesquisa. Na fase de desenvolvimento, a empresa realiza licenciamento de patentes e estabelece parcerias de desenvolvimento em conjunto. Cabe salientar que o conceito de codesenvolvimento ou cooperação em P\&D, utilizado por Chesbrough e Schwartz (2007), significa qualquer relação de trabalho em conjunto entre dois ou mais agentes externos com o objetivo de criar e entregar produtos, tecnologias e serviços.

Entre os principias agentes externos e suas contribuições para os processos de inovação, destacam-se: a) Fornecedores - auxiliam na criação de novos produtos que demandam tecnologias complexas, apresentando um forte impacto nos resultados de inovação (UN, CUERVO-CAZURRA e ASAKAWA, 2010); b) Instituições de ciência e tecnologia (ICTs) - facilitam o acesso a novas pesquisas e a pesquisadores, fontes valiosas para auxiliar na solução de problemas e na geração de inovações (COHEN e LEVINTHAL, 1990); c) Consumidores - contribuem para a redução

\section{Figura 1 - Modelo de inovação aberta}

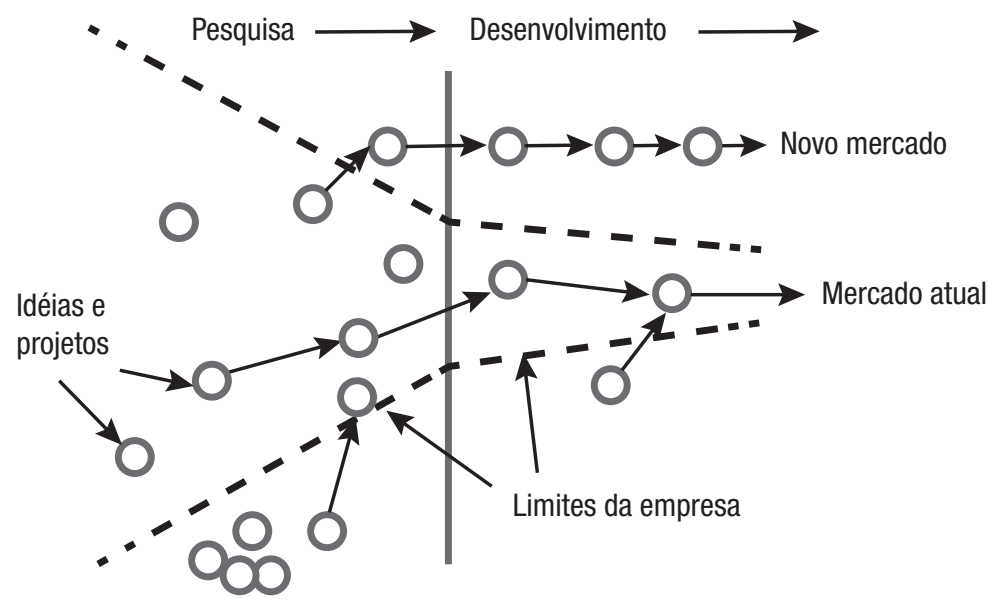

Fonte: Adaptado de CHESBROUGH, 2003. 
de riscos de fracasso de um novo produto no mercado (GASSMANN e ENKEL, 2004), constituindo-se em uma das principais fontes de ideias novas para o processo de P\&D (POETZ e SCHREIER, 2012); d) Competidores - promovem ganhos de escala em pesquisas básicas conjuntas, com a diminuição de custos de P\&D e a combinação de competências únicas de cada empresa (BENGTSSON e KOCK, 1999); e) Intermediários - identificam e conectam atores com interesses comuns, para troca ou comercialização de ideias ou tecnologias desenvolvidas (HOWELLS, 2006; HAMEL e BILL, 2008).

\section{METODOLOGIA DA PESQUISA}

A estratégia de pesquisa neste trabalho foi o estudo de caso, e a unidade de análise foi o projeto Fiat Mio, da empresa Fiat Automóveis, localizada em Betim, MG, Brasil. A seleção do projeto Fiat Mio ocorreu a partir de empresas brasileiras que apresentaram casos no Open Innovation Seminar 2009 - seminário de discussão do tema inovação aberta, ocorrido em São Paulo, em agosto de 2009 - e por se tratar de um caso no qual as práticas de inovação aberta estavam sendo efetivamente empregadas. Com o objetivo de compreender como ocorreram as práticas de inovação colaborativa no âmbito do caso selecionado, a pesquisa de campo foi conduzida à luz de dimensões conceituais e variáveis de análise (Quadro 1). As dimensões incluíram o processo de desenvolvimento de novos produtos, as fontes externas de conhecimento e o tipo de relação estabelecida com agentes externos.
A pesquisa de campo foi conduzida em três etapas, a saber: a) coleta de dados secundários, b) coleta de dados primários e c) análise dos dados. A primeira etapa teve a finalidade de tornar o contexto em que a pesquisa foi conduzida mais familiar ao pesquisador. Para tanto, foram realizados levantamentos de bibliografia empírica sobre o setor automotivo no Brasil, a empresa Fiat e o projeto Fiat Mio. Pesquisaram-se informações em artigos, sites de empresas automotivas e outros materiais publicados em jornais e revistas sobre a indústria. Esses dados deram suporte à construção do instrumento utilizado na coleta de dados.

A segunda etapa consistiu na coleta de dados primários a partir de três técnicas:

a) Análise de documentos - foram coletados dados do site institucional da empresa e da plataforma criada na internet para postagens e discussões de ideias relacionadas ao projeto Fiat Mio. Também foi objeto de análise o blog com informações sobre a P\&D do projeto.

b) Grupo focal - para a compreensão da dinâmica da evolução do projeto, utilizou-se a técnica do grupo focal, com a participação de cinco designers do Centro Estilo da Fiat. O Centro Estilo da fábrica em Betim é o único que desenvolve produtos e tem autonomia para isso fora da Europa. Essa é uma das áreas mais envolvidas no projeto do Fiat Mio, por serem responsáveis por transformar as ideias dos consumidores em conceitos norteadores no desenvolvimento do carro. Por essa razão, optou-se pelo grupo focal com essa área da empresa, o qual ocorreu no segundo semestre de 2010 na Casa Mio, espaço especialmente criado para o desenvolvimento do projeto Fiat Mio. A dinâmica do

\section{Quadro 1 - Dimensões e variáveis de análise}

DIMENSÕES DE ANÁLISE

Processo de desenvolvimento de novos produtos

Fontes externas de conhecimento

Tipo de relação com agentes externos

\section{VARIÁVEIS}

Geração e seleção de ideias; criação de conceito; viabilidade; desenvolvimento; testes; lançamento

Institutos de Ciência e Tecnologia (ICTs); consumidores; fornecedores; intermediários (brokers); concorrentes

Aquisição e licenciamento de tecnologia; cooperação em P\&D; cooperação em gestão de P\&D 
grupo focal foi conduzida pelo pesquisador, que colocava questões para o estímulo do debate, teve duração de duas horas e todas as discussões foram gravadas para posterior transcrição.

c) Entrevistas em profundidade - as entrevistas foram realizadas com especialistas envolvidos no projeto e contou com o auxílio de um questionário semiestruturado, desenvolvido a partir das variáveis do quadro conceitual (Quadro 1). No total, foram realizadas, de maneira presencial, com duração média de uma hora e documentadas por meio de gravações, cinco entrevistas em profundidade, com duas pessoas da engenharia de inovação, duas da pesquisa de mercado e uma da publicidade. Além disso, foram realizadas anotações durante as entrevistas, com o registro de informações relevantes e palavras-chave.

A terceira etapa da pesquisa consistiu na análise dos dados. Todas as entrevistas e a dinâmica do grupo focal foram transcritas e agrupadas em categorias, de acordo com as dimensões e variáveis de análise (Quadro 1). A interpretação dos dados foi realizada de maneira descritiva, com o objetivo de trazer evidências sobre as práticas de inovação colaborativa no contexto do caso em estudo. Deve-se destacar, também, que a escolha das pessoas-chave para as entrevistas em profundidade e para a realização do grupo focal teve como critério os seguintes itens: terem amplo envolvimento no desenvolvimento do projeto Fiat Mio; serem especialistas nas atividades de P\&D do projeto; e apresentarem disponibilidade e concordância para participarem da pesquisa.

\section{RESULTADOS DA PESQUISA}

\section{Descrição do Projeto Fiat Mio}

O centro de P\&D da Fiat no Brasil vem projetando automóveis e avançando tecnologicamente desde 2003. Entre os carros totalmente desenvolvidos no Brasil, estão o Fiat Concept Car I (FCCI) e o Fiat Concept Car II (FCCII), apresentados no Salão Internacional do Automóvel em suas duas últimas edições. Esse evento, que ocorre na cidade de São Paulo, é bianual e apresenta "carros- conceitos", os quais representam o futuro do automóvel em relação a design, engenharia, tecnologia e usabilidade, entre outras características. Para o Salão de 2010, a
Fiat resolveu apresentar um carro inovador não só no conceito mas também no processo de P\&D. Para a criação do FCCIII, a empresa organizou o Fiat Mio, um projeto participativo, no qual os consumidores são convidados a dar ideias para a criação do carro do futuro, por meio de uma plataforma montada na internet para esse fim (fiatmio.cc).

O projeto é diferente dos outros que a Fiat Brasil vem desenvolvendo, especialmente por duas razões: a cocriação com os clientes e a utilização de uma plataforma aberta de inovação. Um dos pilares para o desenvolvimento desse produto é a cocriação entre os consumidores e a Fiat, por meio da postagem de ideias na plataforma fiatmio.cc, as quais são avaliadas e, com base em estudos de viabilidade técnica, transformadas em um componente do automóvel pelos engenheiros e designers. O supervisor de pesquisa de mercado salienta: "É um carro que não tem uma criação exclusiva da Fiat, é algo inovador, pois as pessoas dizem como querem esse carro [...]. Então essa é uma mudança de cultura muito grande". A originalidade do desenvolvimento de um produto por meio de um relacionamento aberto com os clientes é algo não usual em uma indústria para a qual o sigilo dos projetos é a prática habitual. Essa mudança também é enfatizada pelo analista de inovação: "Essa é uma quebra total de paradigma de desenvolvimento em uma fábrica. Guardamos tudo a oito chaves, é segredo e segredo de tudo [...]. A principal diferença é realmente em relação ao que fazemos e esse processo é mais aberto".

A plataforma criada pelo projeto é o que dá suporte a todo o processo, sendo composta por três interfaces de interação com o consumidor: ideias livres, montagem técnica e making of. Cada uma delas tem um papel no projeto e é enfatizada em fases diferentes do desenvolvimento do produto, e todas visam à interação com os agentes externos que participam da construção do carro. Pelo site, o público pode acompanhar toda a criação do veículo, que teve seu desenvolvimento aberto. A interface ideias livres tem ênfase na captação de ideias e no compartilhamento de informações entre os usuários e a empresa. A segunda interface, denominada montagem técnica, foi utilizada para fomentar discussões sobre temas que ainda não tenham sido aprofundados, ou para confirmar as interpretações que os técnicos realizaram das ideias postadas. A terceira interface é o making of, que possibilitou acompanhar o desenvolvimento do carro, abrangendo o trabalho de engenheiros, designers e 
profissionais de outras áreas da empresa envolvidos no projeto Fiat Mio.

Com a utilização de uma plataforma aberta, o resultado final do projeto será de propriedade coletiva. Assim, no momento em que o colaborador posta uma ideia no site, ela deixa de ser sua. Desse modo, o conhecimento produzido poderá ser utilizado por quem desejar, inclusive outras montadoras, conforme mencionado: "A Fiat acredita que o conhecimento gerado neste projeto deve ser propagado sem restrições, podendo ser utilizado por simples usuários ou até mesmo engenheiros e outros fabricantes de veículos" (FIATMIO.CC, 2010). Nem todo o processo de P\&D do projeto, todavia, trabalha com o conceito de patente aberta, segundo afirmação da analista de publicidade: "Como estamos fazendo uma criação coletiva, dividimos o projeto em dois momentos. Um é a criação amplamente coletiva, em que qualquer pessoa pode ter acesso, e o outro é quando a empresa desenvolve novas ideias do carro-conceito a partir da interpretação dos colaboradores". Observa-se que, nesse segundo momento, o que surgir a partir de inovações será de propriedade intelectual da Fiat.

\section{Processo de desenvolvimento do Fiat Mio}

Para a análise do desenvolvimento do projeto FCCIII, foram contempladas quatro fases de desenvolvimento, seguindo as orientações conceituais de Cooper (1990) e Clark e Wheelwright (1993), a saber: (1) mapeamento de cenários, (2) exploração de concept ideas, (3) concept design, que é dividido em duas partes (conceito aberto e conceito da Fiat) e (4) branding.

O mapeamento de cenários foi a fase que deu início ao projeto, ou seja, quando foi tomada a decisão a respeito de qual era a melhor maneira de interagir com o consumidor para envolvê-lo no desenvolvimento do carro. Ao fim desse estágio, optou-se por criar a plataforma de internet fiatmio.cc, com uma pergunta-chave sobre o carro do futuro. A fase exploração de concept ideas constituiu-se na geração de ideias para o produto, as quais foram criadas por meio da plataforma, de modo que as pessoas entravam no site, postavam suas ideias e podiam comentar as ideias dos outros, e inclusive votar nelas, como explica a analista de publicidade: "Inicialmente, coletamos essas ideias e sugestões, inspirando as pessoas com outras ideias que são tendências em outros países e em outras áreas ligadas à tecnologia e ao design".
A fase de concept design foi dividida em duas partes. A primeira, open concept design, visou a interpretar as ideias geradas, sendo a criação coletiva entre a empresa e os consumidores que participaram desse processo criativo. O conceito criado originará uma especificação, que é um documento com propriedade intelectual aberta (creative commons), descrevendo o conceito elaborado para o carro com base no que foi levantado durante essa fase. A segunda parte é o Fiat concept design, que é o desenvolvimento da Fiat. Trata-se da leitura da empresa acerca do conceito que foi criado e que dará origem ao protótipo do FCCIII.

O último estágio, denominado open branding, começa antes da finalização do desenvolvimento do projeto. É nesse estágio que ocorrem as decisões sobre o lançamento do carro. A analista de publicidade esclarece: "No open branding vamos trabalhar a marca, o nome do carro e toda a comunicação também de forma colaborativa [...] para conseguirmos envolver outras pessoas e não só aquelas interessadas em carro". Essa fase está em andamento e tem por objetivo definir a campanha de lançamento, integrando o consumidor mais uma vez por meio das decisões de marketing, divulgação e lançamento.

O projeto Fiat Mio foi criado com o objetivo de desenvolver um carro por meio de um processo aberto e utilizar os consumidores como participantes ativos. O processo de desenvolvimento do FCCIII apresenta a colaboração dos consumidores com maior intensidade nas fases de geração de ideias e lançamento do produto. Além dos consumidores, contudo, a empresa utilizou outras fontes externas de conhecimento para o desenvolvimento desse carro, por meio de diferentes tipos de relação.

\section{Fontes externas de conhecimento}

Embora a participação dos consumidores tenha sido de grande relevância no desenvolvimento do Fiat Mio, outros agentes externos foram acessados em diferentes fases do projeto. O Quadro 2 apresenta algumas das evidências empíricas que demonstram a participação de agentes externos na participação do projeto. De acordo com os entrevistados, as participações mais significativas ocorreram com clientes e fornecedores, havendo também integração com uma universidade.

Os consumidores são vistos no projeto como codesenvolvedores. São eles que tomam todas as deci- 
sões sobre o que o carro deve ou não conter, quais as tecnologias envolvidas, como é o design, entre outras definições. Por outro lado, tornar essas ideias um produto físico, transformando-as em realidade, é função da Fiat, o que segue a linha de Lettl, Herstatt e Gemuenden (2006), para os quais a colaboração dos consumidores é mais intensiva nas primeiras fases do processo. As falas dos entrevistados enfatizam o consumidor como um coparticipante no desenvolvimento do carro, devido à grande interação entre ele e a empresa. De acordo com Enkel, Perez-Freije e Gassmann (2005), Fetterhoff e Voelkel (2006), Dodgson, Gann e Salter (2006), essa interação caracteriza o consumidor como um codesenvolvedor do produto.

A utilização de ICTs no projeto Fiat Mio ocorreu de duas maneiras. No início do projeto, os responsáveis pela área de comunicação da Fiat visitaram universidades e convidaram alunos e professores a participar do projeto por meio da plataforma fiatmio.cc, com a finalidade de atrair ideias de especialistas em áreas relacionadas a automóveis. Com base nisso, uma parte das ideias recebidas foram provenientes de universidades e, tal como os consumidores, elas atuaram como coparticipantes na geração do conceito. Além dessa participação, ocorreu a aquisição de patentes pertencentes a universidades quando a empresa precisava de uma tecnologia que não tinha à sua disposição, fato considerado por Tigre (2006) como uma boa maneira de adquirir tecnologia.

A participação dos fornecedores caminhou na linha de aquisição de tecnologias e passou a ser imprescindível quando as ideias dos consumidores começaram a ser implementadas. A Fiat não possuía muitas das tecnologias requeridas e levantadas no briefing do carro. Como se trata de tecnologias que são muito recentes ou que ainda não são aplicadas em automóveis, a empresa precisou mover esforços para buscar essas tecnologias. Para o desenvolvimento do FCCIII, a Fiat contou com dois tipos de fornecedores: os atuais e as novas empresas especializadas em tecnologia. Quando uma nova tecnologia era requerida, a Fiat buscava primeiramente os fornecedores com os quais já estava acostumada a trabalhar, ou seja, com os quais mantinha laços mais estreitos. Segundo Tidd, Bessant e Pavitt (2001) e Claro (2004), quando existem relações de longo prazo, o desenvolvimento colaborativo é facilitado. Quando os fornecedores mais antigos não dispunham da tecnologia que era necessária, a empresa foi mais longe e começou a fazer contato com empresas, universidades e grupos de pesquisa especializados, os quais poderiam ter o produto desenvolvido ao menos em nível de protóti-

\section{Quadro 2 - Síntese de evidências empíricas do processo de inovação aberta no Fiat Mio}

\section{AGENTE EXTERNO EVIDÊNCIAS EMPIRICAS}

"0 interessante desse projeto é que se trata de um carro realmente cocriado, com a participação de todos [...]" (supervisor de pesquisa de mercado).

Consumidores " "[...] Você transforma o seu consumidor de mero expectador em coparticipante" (analista de inovação). "0 cliente, em vez de passivo, torna-se ativo no desenvolvimento do carro" (especialista em engenharia de inovação).

"0 Fiat Mio era um projeto muito interessante para também levar a marca para dentro das escolas e conseguir parceiros. Então, fomos a universidades ligadas sobretudo às áreas de engenharia e automação, que tinham a ver

ICTs diretamente com o projeto" (analista de publicidade).

"Uma coisa é certa: a academia tem um conhecimento que a indústria não tem, e vice-versa. Deixar esse conhecimento apenas com eles é um erro. Temos de trabalhar criando essa sinergia" (especialista em engenharia de inovação).

"A Click é a principal parceira. Ela formata a informação e envia ao consumidor. [...]. Os profissionais vão passar essa ideia para o nosso consumidor e analisar as respostas" (especialista em engenharia de inovação).

"Estamos fazendo um trabalho de pesquisa, primeiramente com os nossos fornecedores de primeira linha. Quando

Fornecedores ninguém tem essa tecnologia, começamos a buscá-la mais longe" (analista de inovação).

"Há assuntos sobre os quais temos embasamento técnico, sabemos que em algum lugar esses assuntos estão sendo pesquisados, que já foi feito um protótipo ou algo assim [...]. Então vamos atrás para comprar isso" (designer interno). 
po. De acordo com Boehe (2007), essa é uma prática comum em empresas que lidam com componentes de grande complexidade tecnológica. Outra participação de fornecedor no desenvolvimento foi da agência Click, responsável por toda a interação da Fiat com os seus usuários. A empresa participou de todo o projeto de desenvolvimento do FCCIII, sendo inclusive responsável pela execução de uma etapa do processo: a criação do briefing. Por essa razão, a Click é vista por alguns dos entrevistados como a principal parceira no projeto Mio.

Os outros dois agentes externos, todavia, já apontados na literatura, os intermediários (brokers) e os concorrentes, não são utilizados pela Fiat em seus processos de desenvolvimento nem foram utilizados no projeto Fiat Mio. Os entrevistados declararam não utilizar agentes intermediários para acessar conhecimento externo, no entanto, conforme a literatura, os serviços prestados pela agência Click poderiam estar no escopo de um agente intermediário. Quanto aos concorrentes, há apenas colaboração entre empresas de automóveis pertencentes ao Grupo Fiat, como a Ferrari, o que não caracteriza colaboração entre competidores.

\section{Práticas de inovação colaborativa e contribuições para o produto}

A colaboração evidenciada no caso do Fiat Mio vai desde uma parceria para realização de uma atividade de menor complexidade até o desenvolvimento em conjunto de tecnologia entre a empresa e outros agentes. O Quadro 3 sintetiza a dinâmica de desenvolvimento do Fiat Mio, identificando as práticas de inovação colaborativa, as fases do processo de inovação, os agentes externos envolvidos e a contribuição para o produto final. As práticas de inovação colaborativa podem ser agregadas em três grupos: a) aquisição e licenciamento de tecnologia, b) colaboração em P\&D, e c) colaboração em gestão de P\&D.

A aquisição e o licenciamento de tecnologia ocorreram no estágio de desenvolvimento do FCCIII, quando

\section{Quadro 3 - Síntese das práticas de inovação colaborativa no desenvolvimento do Fiat Mio}

\section{PRÁTICAS DE INOVAÇÃO COLABORATIVA}

\section{FASES DO PROCESSO DE INOVAÇÃO}

\section{CONTRIBUIÇÃO PARA} O PRODUTO

Colaboração em P\&D

Colaboração em P\&D

Aquisição e licenciamento de tecnologia e colaboração em P\&D

Aquisição e licenciamento de tecnologia e colaboração em P\&D

Aquisição e licenciamento de tecnologia

Pesquisa - geração e seleção de ideias e criação de conceito

Pesquisa - geração e seleção de ideias e criação de conceito

Desenvolvimento

Desenvolvimento

Desenvolvimento

Fornecedores atuais

ICTs (alunos e professores de universidades)

ICTs (grupos de pesquisa de universidades)

Fornecedores (start-ups e empresas incubadas)

Colaboração em gestão da P\&D
Pesquisa e desenvolvimento
Fornecedores (Agência Click)
Capacidade de 0 automóvel receber atualizações e novas configurações

Novas soluções para carregamento da bateria no carro elétrico

Transmissão de eletricidade sem fio

Tecnologia que possibilite aos vidros mudarem de cor

Componentes para fabricação de carro elétrico, com bateria cambiável

Criação e gerenciamento da plataforma, relação com 0 consumidor, briefing, decisões de conceito 
as ideias estavam tornando-se um produto. Essa prática foi bastante utilizada no projeto Fiat Mio devido ao seu curto tempo de desenvolvimento. Por isso, as ideias geradas no site deveriam estar no carro até o fim do projeto, o que fez com que a Fiat recorresse a detentores do conhecimento necessário e fizesse o licenciamento de sua propriedade. Entre as tecnologias encontradas pela empresa, constam novas formas de transmissão de energia e vidros que modifiquem a sua transparência. Em um primeiro momento, essas tecnologias foram pesquisadas entre os fornecedores atuais, que as disponibilizam para o setor automotivo e para a Fiat. Quando essa tecnologia estava para ser aplicada aos carros, a Fiat procedeu à sua aquisição. A contratação externa de tecnologia, também conhecida como outsourcing para inovação (HUIZINGH, 2011), é indicada por GASSMAN, ENKEL e CHESBROUGH (2010) como a primeira etapa de inovação aberta, avançando em direção a modelos mais estratégicos, como o desenvolvimento em conjunto de P\&D e outras práticas de cocriação.

A colaboração em P\&D ocorreu de maneira ampla com consumidores, fornecedores e universidades. Os consumidores, no entanto, tiveram um nível de envolvimento na cocriação do produto de modo destacado, atuando na geração e seleção de ideias e na criação do conceito do carro. Apresentaram, também, novos conteúdos à empresa, tal como possibilidades de tornar o carro atualizável e capaz de receber novas configurações. Além de gerar ideias e conteúdos, eles foram responsáveis por decidir o caminho do projeto, como no caso da decisão da linha-guia de design do carro, o que, em projetos normais, caberia à direção escolher. No caso do Fiat Mio, as duas linhas-guias de design foram disponibilizadas na plataforma, e o usuário optou por qual delas seria escolhida. A possibilidade de o consumidor influenciar e, em alguns casos, ser envolvido no processo de decisão sobre os caminhos do projeto traz na prática a essência das teorizações de Von Hippel (2005) sobre a necessidade e relevância de maior "democratização" dos processos de inovação.

A colaboração na gestão da P\&D também foi identificada, especialmente na relação com a agência Click, fornecedor de mídia digital da Fiat. Ela participou de todas as etapas do processo de desenvolvimento desde o seu início, sendo responsável por todas as interações com os usuários, pelas postagens na plataforma da internet, pela captação das ideias, pela transformação dessas ideias em briefing e pela avaliação das áreas de criação a partir do que foi requerido no site. Ao falar da participação da Click, um engenheiro explica: "Eles têm um know-how que nós não temos. Então eles estão fazendo o trabalho de juntar todas as ideias e [...] olhar para as nossas criações vendo se é o que as pessoas pediram ou não" (especialista em engenharia de inovação). As evidências salientaram o papel central que a agência Click desempenhou na gestão do relacionamento entre a Fiat e o ambiente externo, exercendo uma função de agente intermediário, conforme conceituação de Howells (2006).

\section{DISCUSSÃO DOS PRINCIPAIS RESULTADOS}

No caso do projeto Fiat Mio - que gerou o desenvolvimento do FCCIII - foram observadas práticas de inovação colaborativa em todos os estágios do processo. Conforme pode ser observado na Figura 2, o desenvolvimento do automóvel foi dividido em cinco fases, sendo que as duas primeiras foram de captação de ideias para o produto, a terceira foi de desenho do conceito, a quarta, de desenvolvimento do protótipo e a quinta, de decisões do lançamento. Em cada uma dessas fases, diferentes agentes externos foram acessados, como consumidores, universidades e fornecedores, permitindo explorar novos conhecimentos, desenvolver novos conceitos e implementar novas tecnologias para o novo produto. Essas evidências estão alinhadas ao trabalho de Reed, Storrud-Barnes e Jessup (2012), indicando que "abrir-se para novas ideias do ambiente externo fortalece a performance de inovação das firmas" (REED, STORRUD-BARNES e JESSUP, 2012, p. 69).

$\mathrm{Na}$ fase em que o protótipo do carro foi desenvolvido, a Fiat precisou de conhecimento especializado para atender a definições relacionadas às tecnologias levantadas pelo briefing. Os resultados de colaboração nessa etapa vão ao encontro das afirmações de Boehe (2007) de que, quando há maior complexidade tecnológica, a relação com fornecedores é maior. A Fiat buscou essas tecnologias primeiramente entre os fornecedores com os quais ela já mantinha contato, adquirindo ou licenciando a patente. Quando esses fornecedores não dispunham da tecnologia necessária, a busca estendeu-se para outras empresas, chegando a start-ups, incubadoras e universidades. Em alguns casos, quando essas tecnologias não eram aplicáveis a veículos, a Fiat auxiliou no desenvolvimento da inovação em conjunto 
com os fornecedores, práticas já muito utilizadas por empresas como a Toyota (DYER e NOBEOKA, 2000) e a BMW (WILHELM, 2011).

Os consumidores tiveram um papel significativo nas fases iniciais de desenvolvimento do FCCIII, auxiliando não só com novos conteúdos, ideias e definições mas também com as decisões de conceito acerca do carro. Essa interação é caracterizada pelo codesenvolvimento e, de acordo com Fetterhoff e Voelkel (2006) e Dodgson, Gann e Salter (2006), o consumidor não é utilizado apenas para pesquisas de mercado e testes de produto, mas ele se torna ativo no desenvolvimento do produto. Especialmente em fases iniciais de P\&D, como no levantamento de ideias e desenvolvimento de conceitos, os consumidores, como usuários, poderão contribuir com aprimoramentos de usabilidade e design que serão fundamentais para o êxito do novo produto no mercado. Já nas fases do desenvolvimento do Fiat Mio, no qual o conhecimento tecnológico foi mais intenso, os consumidores apresentaram uma contribuição menos significativa e a empresa procurou relacionar-se com outros agentes, como empresas start-ups e universidades.

Outro resultado observado foi a redução de tempo de desenvolvimento do produto, conforme já indicado por Chesbrough (2003). No caso do Fiat Mio, a redução do tempo de desenvolvimento foi claramente observada: um projeto que normalmente leva três anos para ir ao mercado ficou pronto em 18 meses. Além das novas tecnologias provenientes do desenvolvimento do FCCIII, as quais a empresa utilizará para desenvolvimentos futuros, o projeto originou uma plataforma de relacionamento com o consumidor que pode ser utilizada não só para futuras pesquisas de mercado e tendências da indústria mas também na identificação de mudanças referentes à opinião desse consumidor.

De modo geral, os resultados do projeto Fiat Mio estão alinhados aos estudos de Dodgson, Gann e Salter (2006) e Van der Meer (2007), os quais visam a compreender como ocorre a interação entre as empresas e o

\section{Figura 2 - Síntese do processo de inovação colaborativa do Fiat Mio}

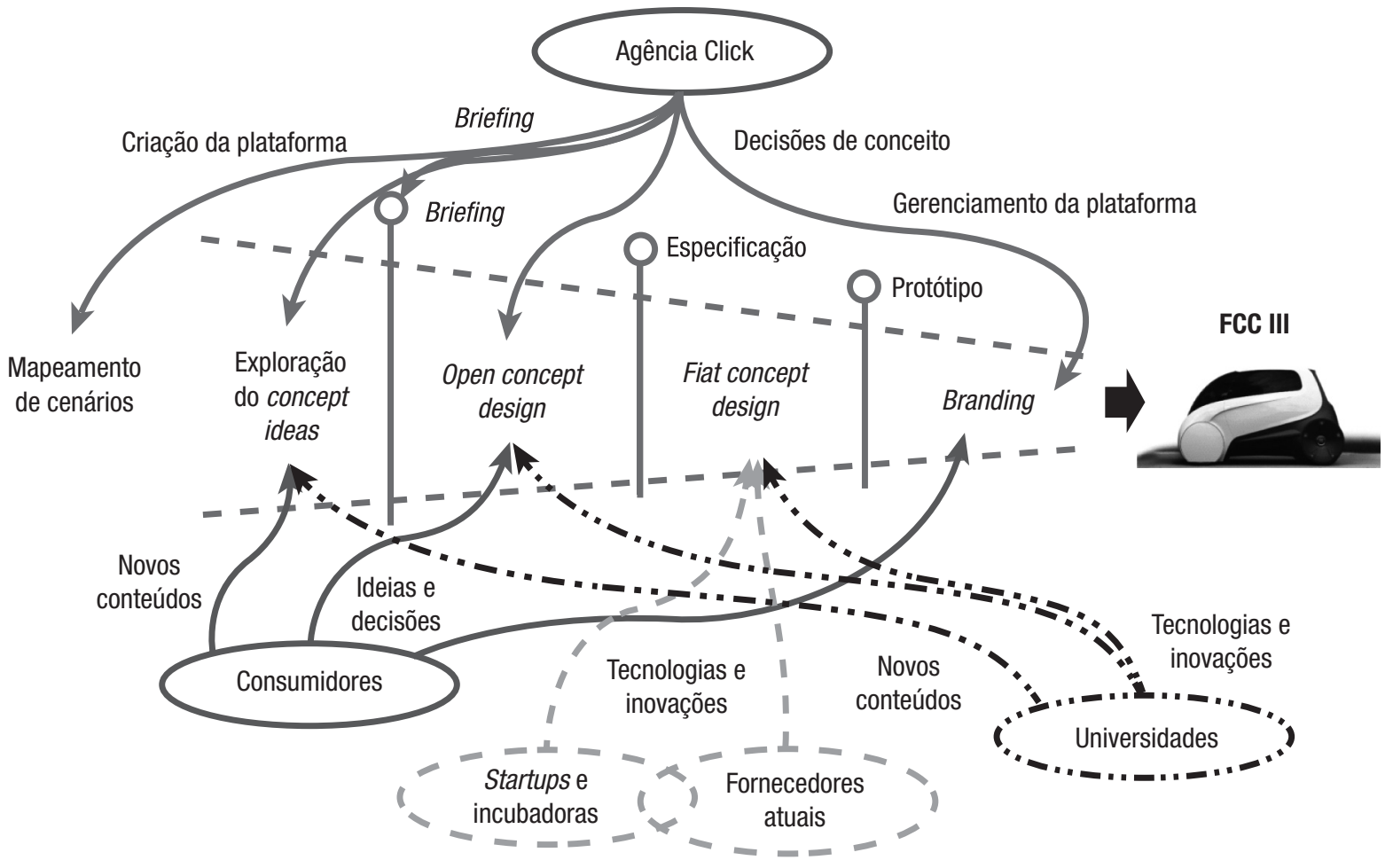


ambiente externo no processo de geração de inovação. A dinâmica de inovação, apresentada na Figura 2, indica que o desenvolvimento do FCCIII ocorreu por meio de um amplo processo de colaboração em rede. A empresa se abriu para o ambiente externo, colocou a sua capacidade de P\&D em sinergia com a inteligência coletiva de milhares de consumidores, complementou com os recursos de universidades e fornecedores e concretizou em forma de um novo produto - um carro conceito.

\section{CONSIDERAÇÕES FINAIS}

As postulações teóricas e as evidências empíricas apresentadas neste estudo visam a contribuir com os crescentes debates acerca do tema de inovação tecnológica. O propósito central do artigo foi de identificar práticas de inovação colaborativa no desenvolvimento de um novo produto, no contexto da indústria automotiva brasileira. Os resultados do estudo de caso permitiram levantar práticas de inovação colaborativa, agrupadas em aquisição e licenciamento de tecnologia, colaboração em P\&D e colaboração em gestão de P\&D. Essas práticas possibilitaram ganhos para o produto final, especialmente no desenvolvimento de novos componentes e de tecnologias, algumas inéditas para a indústria automotiva. As evidências de campo também identificaram três agentes externos relevantes no desenvolvimento do projeto Fiat Mio, entre eles os consumidores, os fornecedores e uma universidade.

A colaboração com fornecedores e universidades, no desenvolvimento de P\&D, já é uma prática utilizada por outras empresas da indústria automotiva. Algo um tanto inédito, especialmente para essa indústria, é a ampla colaboração com o usuário final para atividades de P\&D. Esses achados sinalizam uma mudança, mesmo que ainda experimental, de maior participação do consumidor na cocriação de novos produtos. Outra evidência que chamou a atenção por sua relevância na gestão do processo de P\&D foi o papel exercido pela agência Click em atividades de criação e gerenciamento da plataforma colaborativa de relacionamento com os consumidores. No caso estudado, a agência exerceu a função de broker, intermediando a relação com o consumidor no levantamento de ideias, na cocriação de conceitos e nas decisões sobre itens e componentes para o produto final.

A análise do caso também serviu para trazer novas reflexões sobre como as empresas podem conduzir seus processos de inovação. A transição de um modelo mais fechado, em que o foco recai demasiadamente no desenvolvimento a partir de recursos internos, para um modelo mais aberto, em que as interações com o ambiente externo passam a ser mais frequentes, sinalizam novos caminhos para a P\&D de novos produtos. Os resultados, no entanto, devem ser relativizados, pois o caso descreve o desenvolvimento de um carro-conceito e, como tal, permitiu uma série de experimentações, não representando a realidade dos padrões de desenvolvimento na indústria automotiva. Além disso, um caso idiossincrático é relevante de ser estudado com a finalidade de gerar bases para pesquisas futuras. Um exemplo é analisar as percepções dos agentes externos que participam do processo de desenvolvimento de novos produtos, como consumidores e fornecedores, e compará-las às percepções da empresa para analisar convergências e divergências na maneira como eles perceberam o processo aberto e colaborativo de desenvolvimento do projeto Fiat Mio.

\section{REFERÊNCIAS}

BENGTSSON, M; KOCK, S. Cooperation and competition in relationships between competitors in business networks. Journal of Business E Industrial Marketing, v. 14, n. 3, 1999.

BERTHON, P. R; PITT, L. F; MCCARTHY, I; KATES, S. M. When Customers Get Clever: Managerial Approaches to Dealing with Creative Consumers, Business Horizons, v. 50, n. 1, p. 39-47, 2007.

BOEHE, D. M. Os papéis de subsidiárias brasileiras na estratégia de inovação de empresas multinacionais estrangeiras. RAUSP-Revista de Administração, v. 42, n. 1, p. 5-18, 2007.

CHESBROUGH, H. Open innovation: the new imperative for creating and profiting from technology. Boston, MA: Harvard Business School Publishing, 2003.

CHESBROUGH, H.; VANHAVERBEKE, W; WEST, J. Open Innovation: Researching a New Paradigm. Oxford: Oxford University Press, 2006.

CHESBROUGH, H; SCHWARTZ, K. Innovating business models with co-development partnerships. Research Technology Management, v. 50, n. 1, p. 55-59, 2007. 
CLARK, K. B; FUJIMOTO, T. Product development, performance strategy, organization and management in the world auto industry. Cambridge: Harvard Business Press, 1991.

CLARK, K. B; WHEELWRIGHT, S. C. Managing new product and process development. New York: The Free Press, 1993.

CLARO, D. P. Managing business networks and buyer-supplier relationships. Netherlands: Wageningen University and Research Centre, 2004.

COHEN, W. M; LEVINTHAL, D. A. Absorptive capacity: a new perspective of learning and innovation. Administrative Science Quarterly, v. 35, p. 128-152, 1990.

COOPER, R. Stage gate system a new tool for managing new products. p. 44-53, Business Horizons, 1990.

DYER, J. H; NOBEOKA, K. Creating and managing a high-performance knowledge-sharing network: the Toyota case. Strategic Management Journal, v. 21, n. 3, p. 345-367, 2000.

DEBRESSON, C. Understanding technological change. Montreal: Black Rose Books, 1997.

DITTRICH, K; DUYSTERS, G. Networking as a means to strategy change: the case of open innovation in mobile telephony. Journal of Product Innovation Management, v. 24, p. 510-521, 2007.

DODGSON, M; GANN, D; SALTER, A. The role of technology in the shift towards open innovation: the case of Procter \& Gamble. RED Management, v. 36, n. 3, p. 333-346, 2006.

DOSI, G. Technical change and economic theory. London: Printer, 1988.

ENKEL, E; PEREZ-FREIJE, J; GASSMANN, O. Minimizing market risks through customer integration in new product development: learning from bad practice. Creativity and Innovation Management, v. 14, n. 4, p. 425-437, 2005.

ENKEL, K; GASSMANN, O; CHESBROUGH, H. Open R\&D and open innovation: exploring the phenomenon. $R E D$ Management, v. 39, n. 4, p. 311-416, 2009.

FETTERHOFF, T. J; VOELKEL, D. Managing open innovation in biotechnology. Research Technology Management, v. 49, n. 3, p. 14, 2006.
FIGUEIREDO, P. N. Aprendizagem tecnológica e inovação industrial em economias emergentes: uma breve contribuição para o desenho e implementação de estudos empíricos e estratégias no Brasil. Revista Brasileira de Inovação, v. 3, n. 2, p. 323-361, 2004.

FIATMIO. Fiat Mio, uma união de ideias. Disponível em: http://www.fiatmio.cc/pt/. Acesso em: 12.04.2010.

FREEMAN, C. A Schumpeterian renaissance? SPRU Electronic Working Paper Series, n. 102, jul, 2003.

FUJIMOTO, T; TAKEISHI, A. Automobiles: strategy-based lean production system. Tokyo: University of Tokyo, discussion papers, 2001.

GASSMANN, O; ENKEL, E. Towards a theory of open innovation: three core process archetypes. R\&D Management Conference (RADMA), Lisboa, 2004.

GASSMANN, O; ENKEL, E; CHESBROUGH, H. W. The future of open innovation. RED Management, v. 40, n. 3, p. 213-221, 2010.

HAGE, J; HOLLINGSWORTH, R. A strategy for the analysis of idea innovation networks and institutions. Organization Studies, v. 21, p. 971-1004, 2000.

HAMEL, G; BILL, G. O futuro da administração. Rio de Janeiro: Campus, 2008.

HOWELLS, J. Intermediation and the role of intermediaries in innovation. Research Policy, v. 35, p. 715728, 2006.

HUIZINGH, E. K. R. Open innovation: State of the art and future perspectives. Technovation, v.31, n. 1, p. 2-9, 2011.

HUSTON, L; SAKKAB, N. Connect and Develop: Inside Procter \& Gamble's new model for innovation. Harvard Business Review, v. 84, n. 3, p. 58-66, 2006.

ILI, S; ALBERS, A; MILLER, S. Open Innovation in the Automotive Industry. RED Management, v. 40, n. 3, p. 246255,2010 .

LETTL, C; HERSTATT, C; GEMUENDEN, H. G. Users' contributions to radical innovation: evidence from four cases in the field of medical equipment technology. RED Management, v. 36, n. 3, 2006. 
MOWERY, D. C; ROSENBERG, N. Technology and the pursuit of economic growth. Cambridge: Cambridge University Press, 1989.

NAPOLEONI, C. Il pensiero economico del 900. Turim: Giulio Einaudi Editore, 1963.

O'CONNOR, Gina C. Open, Radical Innovation: Toward an integrated Model in large established firms. In: CHESBROUGH, H; VANHAVERBEKE, W; WEST, J. Open innovation: researching a new paradigm. Oxford: Oxford University, 2006.

POETZ, M; SCHREIER, M. The Value of Crowdsourcing: Can Users Really Compete with Professionals in Generating New Product Ideas? Journal of Product Innovation Management. v. 29, n. 2, p. 245-256, 2012.

POWELL, W. Learning from collaboration: knowledge and networks in the biotechnology and pharmaceutical industries. California Management Review, v. 40, n. 3, p. 228, 1998

POWELL, W; KOPUT, K. W.; SMITH-DOERR, L. Interorganizational collaboration and the locus of innovation: networks of learning in biotechnology. Administrative Science Quarterly, v. 41, p. 116-145, 1996.

REED, R; STORRUD-BARNES, S; JESSUP, L. How open innovation affects the drivers of competitive advantage: Trading the benefits of IP creation and ownership for free invention. Management Decision, v. 50, n. 1, p. 58-73, 2012.

RICHARDSON, G. B. The organization of industry. Economic Journal, v. 82, n. 327, p. 883-896, 1972.

ROTHWELL, R. Industrial innovation: success, strategy, trends. In: DODGSON, M; ROTHWELL, R. The handbook of industrial innovation. Cheltenham: Edward Elgar, 1995.

SCHUMPETER, J. The theory of economic development. Cambridge: Harvard University Press, 1934.

SODA, G. The management of firms' alliance network positioning: Implications for innovation. European Management Journal, v. 29, p. 377- 388, 2011.

TIDD, J; BESSANT, J; PAVITT, K. Managing innovation: integrating technological, market and organizational change. 2. ed. Chichester: John Wiley \& Sons, 2001.
TIGRE, P. B. Gestão da inovação: a economia da tecnologia no Brasil. Rio de Janeiro: Elsevier, 2006.

UN, C. A; CUERVO-CAZURRA, A; ASAKAWA, K. R\&D Collaborations and Product Innovation. Journal of Product Innovation Management, v. 27, n. 5, p. 673-689, 2010.

VAN DER MEER, H. Open innovation - the dutch treat: challenges in thinking in business models. Creativity and Innovation Management, v. 16, n. 2, p. 192-202, 2007.

VON HIPPEL, E. Democratizing innovation. Massachusetts: The MIT Press, 2005.

WILHELM, M. M. Managing coopetition through horizontal supply chain relations: Linking dyadic and network levels of analysis. Journal of Operations Management, v. 29, p. 663-676, 2011. 